\title{
LITERATUR
}

\section{Leadership in der Europäischen Union}

\author{
Joachim Schild*
}

\begin{abstract}
Mit der Auswahl von Herman Van Rompuy und Catherine Ashton als Präsident des Europäischen Rates beziehungsweise als Hohe Vertreterin der Union für Außen- und Sicherheitspolitik haben sich die im Europäischen Rat versammelten Staats- und Regierungschefs jüngst offenbar gegen eine starke politische Führung und gegen die Entstehung eines neuen Kraft- und Machtzentrums innerhalb der Europäischen Union entschieden. Das den neuen, mit dem Vertrag von Lissabon geschaffenen Spitzenämtern zugedachte Rollenprofil scheint dasjenige des Moderators, Koordinators und Konsensschmiedes zu sein. Damit dürften sich die von vielen Beobachtern in die institutionell-vertragsrechtliche Antwort auf das politische Führungsdefizit der Union gesetzten Erwartungen allenfalls teilweise erfüllen. Unter den Mitgliedstaaten war schlicht kein Konsens im Hinblick auf dynamische politische Führung in der Union herzustellen.
\end{abstract}

Gleichwohl dürfte aber eine breite Übereinstimmung unter zentralen europapolitischen Akteuren wie akademischen Beobachtern darüber bestehen, dass politische Führung im Sinne von Orientierung, strategischer Richtungsgebung, Bündelung von Energien und Ausrichtung von Institutionen und Akteuren auf langfristige politische Zielsetzungen sowie die Fähigkeit zur Definition von Prioritäten in der Europäischen Union in jüngerer Zeit zur Mangelware geworden sind.

Von den hier vorzustellenden Publikationen liefern der von Jack Hayward herausgegebene Sammelband „Leaderless Europe“ sowie
Jack Hayward (Hrsg.): Leaderless Europe, Oxford University Press: Oxford/New York 2008, ISBN 978-0-19-953502-6; 336 Seiten, 56,00 £.

Lars Hewel: Hegemonie und Gleichgewicht in der europäischen Integration. Eine Untersuchung der Führungsproblematik im Rahmen der Fortentwicklung der Europäischen Union, Nomos: Baden-Baden 2006, ISBN 978-3-8329-1896-5; 442 Seiten, 69,00 €.

Susanne Pfeiffer: Die deutsch-französische Partnerschaft: störanfällig, aber strapazierfähig? Eine Analyse im Bereich der Außen-, Sicherheits- und Europapolitik (1990-2000), Peter Lang Verlag: Frankfurt Main u.a. 2006, ISBN: 978-3-63154656-7; 419 Seiten, 62,20 €.

Christian Lequesne: La France dans la nouvelle Europe. Assumer le changement d'échelle, Presses de la fondation nationale des sciences politiques: Paris 2008, ISBN 978-2-7246-1085-7; 160 Seiten, 12,00€.

die Dissertation von Lars Hewel einen unmittelbaren Beitrag zur Diskussion über politische Führung in der Union. Die Dissertation von Susanne Pfeiffer zur Entwicklung deutsch-französischer Beziehungen von 1990-2000 sowie die knappe, aber sehr gehaltvolle Monografie von Christian Lequesne zur Entwicklung der französischen Europapolitik seit dem Ende des Ost-West-Konflikts bieten ihrerseits mit der Konzentration auf zwei potenziell führende Mitgliedstaaten in der Union, Deutschland und Frankreich, wichtige ergänzende Erkenntnisse zur Praxis und $\mathrm{zu}$ den Grenzen politischer Führung in der Union. 
Die Europäische Union, so lautet die Kernbotschaft von Hayward in seinem einleitenden Beitrag zu dem von ihm herausgegebenen Sammelband, wird sich auf unbestimmte Zeit darauf einrichten müssen, ohne starke politische Führung auszukommen. Deren Fehlen kann als eine Dauerherausforderung in einer polyzentrischen, machtverteilenden politischen Ordnung wie derjenigen der Europäischen Union, einem komplexen Verhandlungssystem, dessen institutionelle Konfiguration geradezu als antihegemoniale Konstruktion par excellence gilt, betrachtet werden. Aber warum, so fragt Hayward, ist es im Verlauf eines halben Jahrhunderts der Integrationsgeschichte nicht zur Herausbildung einer klar identifizierbaren Form von leadership gekommen? Jean-François Revel zitierend stellt er fest, dass die Antwort möglicherweise die sei, dass Europa die Machtlosigkeit zum Prinzip erhoben habe. „Instead, piecemeal power-sharing by nation statesmen seeks surreptitiously to promote an integrationist strategy incrementally, by fits and starts, and stops", so Hayward. ${ }^{1}$

In Ermangelung eines europäischen Demos erscheint auch die Vorstellung einer prominenten und starken politischen Persönlichkeit an der Spitze der Union als ,simplistisch“, so Hayward. Was unter günstigen historischen Rahmenbedingungen allenfalls möglich erscheint, ist politische Führung ,from behind the scenes", wie sie etwa Jacques Delors gestützt auf ein solides Netzwerk von Vertrauten im fragmentierten EU-System ausüben konnte, zumal er das Vertrauen von François Mitterrand und Helmut Kohl besaß. In dieser Perspektive und Interpretation erscheint der ,Herman Van Rompuy-Typus ' von Spitzenpolitikern geradezu perfekt zum Charakter der Union als Konsensdemokratie zu passen.

Der systematisch angelegte Sammelband behandelt in drei Teilen politische Führung in historischer Perspektive, die Frage, warum es Europa derzeit an politischer Führung mangelt, und schließlich die Frage, woher, insbesondere aus welchen Institutionen - Europäische Kommission, Europäisches Parlament, Europäischer Gerichtshof - heute politische Führungsimpulse kommen könnten. In der Gesamtschau ergibt sich in den meisten Beiträgen ein Bild geringer und tendenziell abnehmender Führungs- oder Steuerungsleistungen, insbesondere was die Rolle der Europäischen Kommission angeht. Sie wird unter anderem in Beiträgen von David Howarth zur Wirtschafts- und Währungsunion und von Michelle Cini behandelt, die den Einflussverfall der Kommission in den Amtszeiten von Jacques Santer und Romano Prodi nachzeichnet.

Politische Führung durch Mitgliedstaaten ist in der Europäischen Union - jenseits der Rolle der Ratspräsidentschaft - allenfalls als kollektive Führung durch eine Gruppe denkbar. Aber Hoffnungen auf kollektive Führung verweist Dionyssis G. Dimitrakopoulos in den Bereich des Wunschdenkens, da drei zentrale Quellen hierfür derzeit fehlten: eine dominante und geteilte Konzeption der materiellen Interessen der Union, ein explizites, legitimierendes und mobilisierendes Set von Ideen und schließlich ein robustes institutionelles Arrangement. $^{2}$

Der Sammelband kann mit einer Reihe von empirisch gehaltvollen Einzelbeiträgen aufwarten. Was er jedoch vermissen lässt, ist die systematische Entfaltung des Begriffs der politischen Führung im europäischen Mehrebenensystem sowie eine theoretische Anstrengung, Bedingungen und förderliche Kontextfaktoren für - zumindest temporäre politische Führungsleistungen herauszuarbeiten. Dies wird nur ansatzweise in den einzelnen Beiträgen geleistet, deren Erkenntnisse aber nicht theoretisch gebündelt werden. Le-

1 Jack Hayward: Introduction: Inhibited Consensual Leadership within an Interdependent Confederal Europe, in: Jack Hayward (Hrsg.): Leaderless Europe, Oxford/New York 2008, S. 1-14, hier S. 1.

2 Dionyssis G. Dimitrakopoulos: Collective Leadership in Leaderless Europe: A Sceptical View, in: Jack Hayward (Hrsg.): Leaderless Europe, Oxford/New York 2008, S. 288-304, hier S. 288. 
diglich der Beitrag von Howarth unternimmt einige Schritte in Richtung einer analytischen Klärung des Begriffs politischer Führung, indem er in Bezug auf die Kommission zwischen unterschiedlichen Manifestationen beziehungsweise Teildimensionen politischer Führung in Form von entrepreneurship, Präferenzmanipulation, Management, Wachhundfunktion und formeller/informeller AgendaSetting-Macht unterscheidet. ${ }^{3}$ Eine Leerstelle des Sammelbandes besteht auch darin, dass der Europäische Rat, der laut Vertrag ,die allgemeinen politischen Zielvorstellungen und Prioritäten“ der Union festlegen und ihr „die für ihre Entwicklung erforderlichen Impulse“ geben soll (Art. $15 \mathrm{EUV}^{4}$ ), dem also die Wahrnehmung von Führungsfunktionen als Rolle und Aufgabe zugewiesen wurde, nicht in einem eigenen Beitrag behandelt wird.

Gleich zwei Beiträge des Bandes befassen sich in kritischer Absicht mit der Führungsrolle deutsch-französischer Beziehungen in der Union. William E. Paterson kommt in seinem historischen Überblick zu dem Ergebnis, dass intergouvernementale Führung durch Mitgliedstaaten, wenn sie denn zu beobachten war, zwar in der Regel gemeinsam von Deutschland und Frankreich ausgeübt wurde; diese sei aber allenfalls sporadisch und unter kontingenten Bedingungen zustande gekommen und werde rückblickend geradezu in mythenbildender Weise verklärt. Das Bild einer „kooperativen Hegemonie“, wie es von Thomas Pedersen ${ }^{5}$ gezeichnet wurde, ist allenfalls in einzelnen Perioden, etwa in Bezug auf historische Richtungsentscheidungen auf dem Weg zur Währungsunion empirisch haltbar. Im Übrigen verweist Paterson auf die mehrfache Bremserrolle des „Tandems“ und auf deutsche „Führungsvermeidungsreflexe“ .6
Alistair Cole sieht seinerseits seit den 1990er Jahren eine Entwicklung hin zu einer eher defensiven, stärker an geteilten nationalen Interessen denn an europäischen Integrationszielen ausgerichtete Rolle beider Länder, was an Entscheidungen zur Gemeinsamen Agrarpolitik sowie insbesondere an der von beiden Staaten gemeinsam betriebenen Verwässerung und Reform des Stabilitäts- und Wachstumspaktes festgemacht werden kann.

$\mathrm{Zu}$ ähnlichen Schlüssen kommen auch die beiden Dissertationen von Hewel sowie von Pfeiffer. Hewel analysiert die Führungsleistungen Frankreichs, Deutschlands und Großbritanniens im Rahmen der jüngeren Integrationsgeschichte nach 1990 in einer strikt neorealistischen Perspektive. Damit nimmt er gewiss eine theoretische Außenseiterposition ein. Diese ist dort fruchtbar, wo im Rahmen der im Zentrum der Arbeit stehenden Vertragsrevisionen von ,Maastricht" bis ,Nizza plausibel dargelegt werden kann, wie machtpolitische Erwägungen und wahrgenommene Verschiebungen der Binnenbalance der Union zu wichtigen Handlungsmotiven für nationale Regierungen und ihr Spitzenpersonal wurden. Empirisch wird dem Leser zwar kaum grundsätzlich Neues zu den Vertragsverhandlungen geboten, die Bedeutung machtpolitischer Interessen für diese Verhandlungsprozesse wird aber durchaus plausibel und gut belegt herausgearbeitet. Angesichts einer zunehmenden Zahl von Veröffentlichungen, die mit hohem theoretischen Aufwand nach noch so kleinen Spurenelementen von Deliberation in der Weiterentwicklung der Europäischen Union fahnden und dabei machtpolitische Interessen aus der Analyse weitgehend ausblenden, kann diese Perspektive durchaus als be-

3 David Howarth: Delegation and Commission Leadership in Economic and Monetary Union, in: Jack Hayward (Hrsg.): Leaderless Europe, Oxford/New York 2008, S. 28-46, hier S. 32.

4 Vertrag über die Europäische Union in der konsolidierten Fassung des Vertrags von Lissabon, in: Amtsblatt der EU, Nr. C 115 vom 9. Mai 2008, S. 13-45.

5 Thomas Pedersen: Germany, France and the Integration of Europe. A realist interpretation, London 1998; Thomas Pedersen: Cooperative hegemony: power, ideas and institutions in regional integration, in: Review of International Studies 4/2002, S. 677-696.

6 William E. Paterson: Did France and Germany Lead Europe? A Retrospect, in: Jack Hayward (Hrsg.): Leaderless Europe, Oxford/New York 2008, S. 89-112, hier S. 99. 
reichernd und als nützliches Korrektiv empfunden werden.

Allerdings erscheint das Erkenntnispotenzial eines neorealistischen Zugriffs auf das Thema durchaus in einem doppelten Sinne begrenzt. Zum einen lassen sich im Rahmen dieser machttheoretischen Perspektive in erster Linie ,epochemachende“ Entscheidungen im Kontext von Vertragsreformen erhellen, kaum aber das allägliche Funktionieren und die konkrete Politikgestaltung im Rahmen des europäischen Mehrebenensystems. Auch ist die Darstellung vor allem dort überzeugend, wo es um Verhandlungsgegenstände geht, die unmittelbar die Machtinteressen der Mitgliedstaaten berühren, vor allem im Bereich der Reform der Institutionen, etwa der Abstimmungsmodalitäten und der Stimmenwägung im Rat. Wesentlich weniger gut kann der neorealistische Ansatz die differenzierte Form der Integrationsentwicklung auf unterschiedlichen Politikfeldern erklären, insbesondere die nur langsamen Fortschritte der Europäischen Union auf dem Wege zu einem globalen Akteur, die schwer mit dem Argument des externen balancing gegenüber den USA in Einklang zu bringen sind.

Auffällig ist, dass der Autor sich praktisch ausschließlich in einem neorealistischen Bezugsrahmen bewegt, ohne konkurrierende Erklärungsansätze überhaupt ernsthaft in Betracht $\mathrm{zu}$ ziehen, seien es nun der liberale Intergouvernementalismus oder institutionalistische Ansätze. Die Systematik und theoretische Stringenz geht hier auf Kosten der Erklärungs- und Prognosekraft. So lautet eine zentrale Schlussfolgerung des Autors, dass die „Entwicklung der Integration insgesamt progressiv [verlaufe]. Den auf die Europäische Union wirkenden systemischen und subsystemischen Rahmenbedingungen ist eine Tendenz zur Vereinheitlichung inhärent, die die Mitgliedstaaten dazu bewegt, interne Ungleichgewichte integrativ abzumildern und ihre Capabilities gegenüber exogenen Herausforderungen zusammenzuführen."7 $\mathrm{Zu}$ einem solch integrationsoptimistischen Ausblick kann nur gelangen, wer nicht nur die wachsende Heterogenität der erweiterten Europäischen Union, sondern vor allem auch die innerstaatlichen und innergesellschaftlichen Widerstände gegen Integrationsvertiefung völlig ausblendet.

Während Hewel Beispiele erfolgreicher deutsch-französischer Führung behandelt, aber durchaus auch machtpolitisch motivierte Konflikte im Kontext der Institutionenreform in den Verhandlungen über die Verträge von Amsterdam und Nizza analysiert, konzentriert sich Pfeiffer in ihrer Studie ganz auf die Art und Weise, wie in den deutsch-französischen Beziehungen bilaterale Konflikte und Spannungen verarbeitet werden. Sie fragt nach der Lern- und Anpassungsfähigkeit der deutschen und französischen Regierungseliten in Anbetracht von Konflikterfahrungen im bilateralen Verhältnis nach der Zeitenwende von 198991 bis ins Jahr 2000. Die Fallstudien sind daher so ausgewählt, dass der Umgang mit bilateralen Divergenzen analysiert und Bedingungen für erfolgreiche Konfliktbearbeitung identifiziert werden können: der Umgang mit dem Jugoslawienkonflikt 1991-1999, Divergenzen in der Sicherheits- und Verteidigungspolitik (Einsatz- und Befehlsstruktur des Eurokorps, französische Armeereform, europäische schnelle Eingreiftruppe) sowie institutionelle EU-Reformen von ,Maastricht' bis ,Nizza‘ am Beispiel der Sitz- und Stimmenverteilung im Europäischen Parlament beziehungsweise im Rat bilden die drei Themenschwerpunkte.

Die Fähigkeit, aus bilateralen Spannungen und Konflikten zu lernen, ist der Autorin zufolge durchaus begrenzt. Sie sieht in den 1990er Jahren einen schleichenden Prozess der Erosion deutsch-französischer Beziehungen und eine Relativierung ihres Charakters

7 Lars Hewel: Hegemonie und Gleichgewicht in der europäischen Integration. Eine Untersuchung der Führungsproblematik im Rahmen der Fortentwicklung der Europäischen Union, Baden-Baden 2006, S. 399. 
als privilegierte Sonderbeziehung. Einen wesentlichen Grund hierfür findet sie im „Streben beider Staaten nach Durchsetzung ihrer Interessen $[\ldots]$, das nicht die Verbesserung des beiderseitigen Zusammenwirkens, sondern das Erreichen der jeweiligen Ziele zum entscheidenden Kriterium nationalen Lernens macht."8

Der Wert der Studie liegt im detaillierten Nachzeichnen bilateraler Konflikte und ihrer Bearbeitung in der Außen-, Sicherheits- und Europapolitik. Die Autorin scheint dabei jedoch Opfer ihrer eigenen Analysestrategie zu werden. Ihre Konzentration auf konflikthafte Prozesse lässt sie einen pessimistisch-alarmistischen Grundton anschlagen und verstellt ihr den Blick auf die Gesamtkonfiguration deutsch-französischer Beziehungen in europapolitischen Fragen in der behandelten Periode sowie auf die zumindest partiell seit 2000 reaktivierte europapolitische Führungsrolle, etwa im Rahmen der Verhandlungen über einen Vertrag über eine Verfassung für Europa.

Die mangelnde Prognosefähigkeit beruht im Falle der vorliegenden Studie auf einer nicht hinreichenden Berücksichtigung des normativen Unterfutters der deutsch-französischen Beziehungen und der damit verbundenen Rollenzuschreibung als führende Staaten in der Union, denen eine herausragende Verantwortung für den Integrationsprozess zukommt. Im Übrigen ist gerade in der jüngsten Zeit zu beobachten, dass eine nüchtern-rationale Interessenabwägung zu dem Schluss führen kann, dass eine führende Rolle in der Europäischen Union nicht ohne oder gegen, sondern nur mit dem privilegierten Partner möglich ist. Diese Erfahrung musste Präsident Nicolas Sarkozy am Beispiel seines Mittelmeerunionsprojekts auf schmerzliche Weise machen.

Sarkozys erklärtes Ziel als Staatspräsident war und ist es, Frankreich seinen Platz als führender Mitgliedstaat im Zentrum der Euro- päischen Union zurückzugeben. Wie und warum dieser nach der Zeitenwende 1989-91 verloren ging, ist zentraler Gegenstand der kompakten, aber äußerst anregenden Studie von Lequesne, dem derzeitigen Direktor des „Centre d'études et de recherches internationales" (Sciences Po Paris). Seine zentrale These lautet, dass Frankreich seinen Platz im erweiterten Europa noch nicht habe finden können, da es die nötigen internen und europapolitischen Anpassungen an die veränderten Realitäten einer erweiterten Europäischen Union (noch) nicht vollzogen habe.

Die 1980er Jahre werden von Lequesne als ein Jahrzehnt des ,ruhigen Funktionalismus“ beschrieben, gekennzeichnet durch eine Abfolge von konkreten Integrationsprojekten, eine von Frankreich gemeinsam mit Deutschland ausgeübte Führungsrolle vor dem Hintergrund eines permissiven Konsenses der Bürger. Das Jahr 1989 bilde eine Zäsur und stehe am Beginn einer starken Verunsicherung der politischen und administrativen Eliten, aber auch der Bürger, einer Desillusionierung im Hinblick auf die Entwicklungsrichtung der Union und auf den möglichen Einfluss Frankreichs im europäischen Rahmen. ${ }^{9}$ Insbesondere der Prozess der Erweiterung wurde stärker als Bedrohung denn als Chance begriffen, eine Bedrohung des französischen Wirtschafts- und Sozialmodells durch verstärkte wirtschaftliche Konkurrenz, zunehmenden Steuerwettbewerb und sozialregulative Unterbietungswettläufe. In einer erweiterten Union schwinden die Realisierungschancen einer ,Europe puissance'-Konzeption aufgrund der ausgeprägt pro-atlantischen Orientierungen der neuen Mitgliedstaaten und droht allgemein ein Einflussverlust eines politisch-voluntaristischen Verständnisses des Integrationsprojekts zugunsten der Wahrnehmung der Union primär als Wirtschaftsgemeinschaft. Damit sinken auch die Chancen, französische Präferenzen auf die europäische Ebene ,hoch-

8 Susanne Pfeiffer: Die deutsch-französische Partnerschaft: störanfällig, aber strapazierfähig? Eine Analyse im Bereich der Außen-, Sicherheits- und Europapolitik (1990-2000), Frankfurt Main u.a. 2006, S. 352.

9 Christian Lequesne: La France dans la nouvelle Europe. Assumer le changement d'échelle, Paris 2008, S. 37. 
zuladen“, während gleichzeitig identitätsstiftende Elemente des nationalen Wirtschaftsund Sozialmodells unter erheblichen Anpassungsdruck geraten. Damit droht die gesellschaftliche Legitimationsgrundlage der französischen Europapolitik wie auch der Europäisierung des nationalen politischen Systems zu erodieren. Vor diesem Hintergrund kam es zu einer Art Defensivkoalition aus föderalistisch orientierten Kleineuropaanhängern, die eine Minderheitenposition einnehmen, und den von Sozialmodellängsten umgetriebenen Europaskeptikern, die in ihrer tief sitzenden Erweiterungsskepsis geeint waren. Die Europaskepsis letzterer war ausschlaggebend für den negativen Ausgang des Verfassungsreferendums vom 29. Mai 2005.

Sozialmodellängste, so Lequesne, sind nicht nur Ausdruck von Globalisierungsängsten, die auf die EU-Osterweiterung projiziert werden, sondern sitzen tiefer: Sie beruhen auf einer generellen Skepsis gegenüber dem Markt als ökonomische Organisationsform und Koordinierungsmechanismus. Die verbreitete Wahrnehmung der Europäischen Union als „Markteuropa“ und als Bedrohung des französischen Wohlfahrtsstaatsmodells verstelle letztendlich den Blick auf endogene Ursachen von sozialen und wirtschaftlichen Fehlentwicklungen im Lande.

Um eine positive, gestaltende Führungsrolle in Europa wiederzuerlangen muss Frankreich, Lequesne zufolge, seinen Frieden mit den Gegebenheiten eines erweiterten Europas und der Globalisierung schließen, intern die nötigen Anpassungsleistungen erbringen und extern nicht lediglich auf die Führung durch große Mitgliedstaaten setzen, sei sie deutschfranzösisch oder franko-britisch, sondern auch mit kleinen und mittleren Staaten zu kooperieren lernen.

Politische Führung in der Europäischen Union, so die gemeinsame Erkenntnis der vorgestellten Studien, ist äußerst voraussetzungsvoll und an eine Reihe von Kontextfaktoren geknüpft, deren Zusammentreffen in der Integrationsgeschichte gewiss nicht der Regelfall war. Kollektive Führung durch Institutionen und/oder Gruppen von Mitgliedstaaten kann in der erweiterten Union gleichwohl als Voraussetzung allein schon für die Bewahrung des erreichten Integrationsstandes in Zeiten wirtschaftlicher Krisen, zunehmend heterogener Interessen und Leitbilder sowie angesichts eines verbreiteten gesellschaftlichen Euroskeptizismus betrachtet werden.

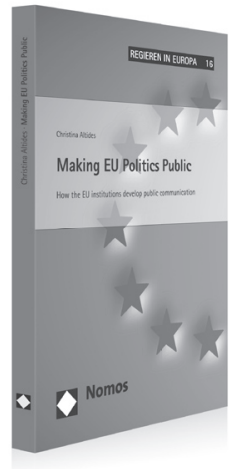

\section{Making EU Politics Public} How the EU institutions develop public communication Von Dr. Christina Altides

2009, 227 S., brosch., 29,- $€$, ISBN 978-3-8329-4966-2

(Regieren in Europa, Bd. 16)

Bitte bestellen Sie im Buchhandel oder versandkostenfrei unter $\downarrow$ www.nomos-shop.de 